\title{
The Effects of Neuromuscular Electrical Stimulation on Pharyngeal Transit Time
}

\author{
Seon-Rye Kim PhD ${ }^{1)}$, Ki-Hyun Kwon, PT, MS ${ }^{2)}$, Byung-Jun Cho, PhD ${ }^{3)^{*}}$ \\ 1) Department of Public Health, Chungnam National University \\ 2) Occupational Therapy Section, Soonchunhyang University Chunan Hospital \\ 3) Department of Emergency Medical Technology, Kangwon National University: Kuydong, Samchuock \\ City, Kangwondo 245-711, South Korea. TEL: +82 33-570-6440
}

\begin{abstract}
Purpose] The purpose of this study was to identify the effects of Neuromuscular Electrical Stimulation (NMES) on pharyngeal transit time. [Subjects] Subjects were divided into normal old persons aged over 70 $(\mathrm{N}=18)$, and normal young persons aged under $30(\mathrm{~N}=10)$. [Method] We measured the Pharyngeal Transit Time (PTT) to determine their swallowing characteristics. NMES was used to stimulate old group's suprahyoid and infrahyoid muscles 60 minutes a day, 5 days a week for 2 weeks using four electrodes. We used SPSS version 14.0 to analyze and compare the data between the 2 groups. [Results] After NMES, PTT of the normal old group became significantly shorter. PTT was much shorter, but was still longer than that of the normal young group. [Conclusion] The PTTs of the two groups classified by age were significantly different.

Key words: Pharyngeal, Video fluoroscopy, Electrical stimulation
\end{abstract}

(This article was submitted Feb. 4, 2013, and was accepted Mar. 8, 2013)

\section{INTRODUCTION}

A normal swallowing function is one of the utmost desires of a human being, satisfying the appetite and protecting the respiratory tract. If there is any problem with this function, it can seriously impair quality of life, and even cause death $^{1)}$. Dysphagia is a problem which curves transporting food from the mouth to the stomach, Recently, its definition has been expanded to cover all swallowing-related behaviors and senses, and preliminary behaviors preparatory to swallowing ${ }^{2}$. Dysphagia mainly occurs in the case of progressive neurological disorders, strokes, cancers of the head and the neck, and in old persons. It is reported that dysphagia occurs in half of the persons who dwell in facilities for the elderly ${ }^{3}$. There have been few studies of the swallowing function of normal old Furthermore, among the studies of the of swallowing function of normal persons, most studies have examined the upward displacement or the anterior displacement of the hyoid bone, and few studies have examined the velocity of swallowing and delay time. Most previous studies of swallowing function were conducted with young adults as subjects. The swallowing process is activated by a complex combination of several structures ${ }^{4,5)}$. Dysphagia primarily or secondarily accompanies many disorders which occur in old persons. Thus, it is highly meaningful to conduct a comparative study of the characteristics of the swallowing function and symptoms of old persons. This study examined the characteristic of swallowing function and PTT of normal old persons aged over

*To whom correspondence should be addressed. E-mail: Cho6451@gmail.com
70 , and compared them with those of a young adult group in order to understand the characteristic of dysphagia due to natural aging. Our aim was to establish research data for the prescription of diet types and program development to enhance swallowing function of old persons.

In this study, we to investigated the characteristics of the swallowing function of normal old persons and compared the functional dysphagia criteria after performing neuromuscular electrical stimulations (NMES) to neck. We performed videofluoroscopic examination of swallowing (VFS) of a soft blended diet (SBD) by a group of normal persons aged over 70 who had no record of swallowing function disorder, and a group of young adults aged under 30. This study aimed to the characteristics of swallowing due to natural aging, and to provide basic data for a rehabilitation program promoting the swallowing function of aged persons.

\section{SUBJECTS AND METHODS}

\section{Subjects}

Subjects were divided into normal aged persons aged over 70 and normal young persons younger than 30 . The normal aged group was comprised of 8 men and 10 women, and thenormal young aged group was comprised of 2 men and 8 women.

The PTT of each group was evaluated using video fluoroscopy; the normal aged group's swallowing characteristics were also evaluated. The PTT of the aged group was evaluated with second video fluoroscopy after NMES. NMES was performed for 60 minutes a day, 5 days a week for 2 weeks on the aged group's suprahyoid and infrahyoid 
muscles using four electrodes. We analyzed the data of the different groups before and after NMES.

None of the subjects had dysphagia and we excluded those who had medical histories of the disorders of central nerve system, muscular diseases, head-neck cancer or esophageal disorders. Those who had cardiac disorder, dermatologic diseases seizures which might have been aggravated NMES were alsoexcluded.

\section{Methods and Procedure}

All subjects understood the purpose of the study and the experimental process. They had read the instructions for subjects and the agreement issued and signed the agreement. The general characteristics of the research subjects are shown in Table 1 . The normal aged persons group $(n=18)$ and the young adult group $(\mathrm{n}=10)$ swallowed $10 \mathrm{~mL}$ of plain yoghurt, which was mixed with barium, three times while receiving VFS in the VFS room of the Department of Radiology in $\mathrm{S}$ hospital in $\mathrm{C}$ city. The examination process was performed by a rehabilitation specialist and a professional. The videotaping was done by a radiologist and recording was done by an intern of the department of rehabilitation. Following examination, the recorded video fluoroscopy images of swallowing were graded using the functional dysphagia scale (FDS $)^{6}$, and the pharyngeal transit time (PTT) of each subject was calculated using a video analysis program.

To observe silent aspiration, VFS was conducted, for the two groups. In the VFS, we observed the process of swallowing a plain yoghurt with a barium contrast medium (Solotop 35\%, Taejoon Pharmacy, Korea), Omnidiagnost Eleva (Philips, Netherlands), and the VFS image was recorded by a S-VHS video recorder. The recorded image was digitized, and analyzed at 30 frames per second using NMES Windows Movie Maker (Microsoft, US). The analysis was conducted, twice before and after the. The examination was conducted by a rehabilitation specialist and a professional in the VFS room of the Department of Radiology, with a subjects swallowing $10 \mathrm{~mL}$ of plain yoghurt mixed with barium three times, while VFS taping was conducted. An electrical stimulation system (Stim Plus DP-2000, Korea) for correcting the process of swallowing was used to treat the 18 members of in the old person group. It is portable two channels, so 4 electrode patches can be placed on two parts. The electrical stimulation used in this study was an $80 \mathrm{~Hz}$, $300 \mu \mathrm{sec}$, bi-phasic waves. The rise and fall time were set to one second for 50 seconds before and after impulse, and a sporadic impulse which was accompanied by 8 seconds of interval time was conducted ${ }^{5)}$. The electrical stimulation intensity started at $3 \mathrm{~mA}$ and was raised until subjects felt gripping in the neck.

The current was maintained at this level with the consent of the subjects. If a subject complained of severe pain or uncomfortable feeling during stimulation, the electrical stimulation was stopped. The subject was educated to perform dry swallowing while receiving electrical stimulation. Moreover, to reduce tension, the subject was allowed to talk freely while participating in the experiment. An occupational therapist who had been trained in electrical stimula- tion therapy of the nerve roots conducted the experiment. The stimulation was conducted 60 minutes a day, 5 times a week for 2 weeks, a total of 10 times.

In this study, some subjects expressed anxiety about the electrical stimulation while receiving NMES the neck muscle, but no subject complained of pain. The subjects were encouraged to visit the clinic of the hospital on time, and to participate in the experiment fully under the supervision of an experimenter. The electrodes were circular pads $20 \mathrm{~mm}$ in diameter. They were attached to two parts of the hyoid bone and two parts of the thyroid notch, to stimulate the digastric muscle, thyreohyoid muscle and general hyoid bone muscles above and below the hyoid bone.

The independent t-test was used to compare between the two groups. Significance was accepted for values of $\mathrm{p} \leq 0.5$.

\section{RESULTS}

Table 2 displays the results of the paired sample t-test regarding PTT before and after NMES of at the neck muscles of the normal old person group. The average value of poststimulation PTT was significantly shorter than the average value of pre-stimulation PTT $(\mathrm{p}<0.05)$.

Table 3 presents the results of the independent sample t-test regarding PTT between the young adult group and the normal old person group after NMES of the neck muscles. The average PTT of the normal old person group sharply decreased after NMES of the neck muscles, and showed a shorter time than the average PTT of the young adult group. The average PTT of the normal old person group reached a level similar to that of the young adult group, but was not significantly different from it ( $p>0.05)$.

Table 1. Characteristics of the subjects

\begin{tabular}{cccc}
\hline \multirow{2}{*}{ Group } & Age & \multicolumn{2}{c}{ Sex } \\
\cline { 2 - 4 } & Mean \pm SD & male & female \\
\hline $\begin{array}{c}\text { the old } \\
\mathrm{n}=18\end{array}$ & $76.2 \pm 4.28$ & $8(44 \%)$ & $10(56 \%)$ \\
$\begin{array}{c}\text { the young } \\
\mathrm{n}=10\end{array}$ & $21.2 \pm 0.42$ & $2(20 \%)$ & $8(80 \%)$ \\
\hline
\end{tabular}

Table 2. PTT pre and post NMES

\begin{tabular}{lccc}
\hline & & $\mathrm{N}$ & Mean \pm SD \\
\hline \multirow{2}{*}{ PTT } & pre & 18 & $0.87 \pm 0.13$ \\
& post & 18 & $0.69 \pm 0.11^{* *}$ \\
\hline
\end{tabular}

PTT: Pharyngeal Transit Time Note. Values are mean \pm SD. * * Significantly different $(\mathrm{p}<0.01)$

Table 3. PTT of each group before NMES

\begin{tabular}{cccc}
\hline & Group & $\mathrm{N}$ & Mean \pm SD \\
\hline \multirow{2}{*}{ PTT } & the old & 18 & $0.69 \pm 0.11$ \\
& the young & 10 & $0.57 \pm 0.04^{*}$ \\
\hline
\end{tabular}

Values are mean \pm SD. $* *$ Significantly different $(\mathrm{p}<0.05)$ 


\section{DISCUSSION}

Neuromuscular electrical stimulation was applied for 60 minutes a day, 5 days a week for 2 weeks to the neck muscles of the normal old person group who showed weaker function in every evaluation item of FDS than the young adult group. The FDS was used to determine the change of swallowing function in the normal old person group induced by NMES of the neck muscles. The paired sample t-test was used to test the differences in the pre and post-electrical NMES FDS values. After 2 weeks of NMES of the neck muscles, the average value of FDS of the old person group had very significantly decreased $(p=0.001)$. The effect of NMES has been demonstrated in many previous studies ${ }^{7-9}$ ) of patients with stroke. NMES of the neck muscles is considered to reverse the functional decrease caused by natural aging of normal old persons. Theory indicates that NMES has a muscle retraining effect, electrically stimulating the nerve muscles ${ }^{10,11)}$. In the individual evaluation items of FDS of the normal old person group after electric stimulation, bolus formation function was $25 \%$ and remainders in the mouth was $44 \%$ at the oral process step. In the pharynx step, remainders in the valleculae was $27 \%$, remainders the pyriform sinus was $19 \%$, and the subject with a problem of barium coating on the pharyngeal wall was $11 \%$. In particular, the delay of pharyngeal swallowing reflection in the pharynx step was $5.6 \%$ before NMES, but no subject who was awarded points after NMES. Functional improvement was achieved in all items of FDS after NMES.

In the FDS evaluation items of the normal old person group after NMES, bolus formation function and remainders in the mouth at the oral process step were significantly improved compared to before NMES, $\mathrm{p}=0.008$ and $\mathrm{p}=0.006$, respectively. Remainders in the valleculae after swallowing and the pyriform sinus after swallowing at the pharynx process step were also significantly improved compared to before NMES, $\mathrm{p}=0.015$ and $\mathrm{p}=0.008$, respectively. Thus, statistically significant improvements were found in 4 out of the 6 evaluation items of FDS. However, in the delay of pharyngeal swallowing and barium coating of the pharyngeal wall after swallowing, no statistically significant differences were found. In the comparisonFDS with the young adult group, in order to compare the degree of improvement of swallowing function after NMES of the neck muscle, the average value $(5.08 \pm 6.8)$ of FDS of the normal old person group after NMES and the average value $(5.3 \pm 6.0)$ of FDS of the young adult group were similar, with no significant difference between them $(p=0.934)$. It can be inferred that the swallowing function of the normal old person group NMES the neck muscles was similar to the function of the young adult group.

\section{REFERENCES}

1) Logemann JA: A manual for videofluoroscopic evaluation of swallowing (2nd ed). Austin:PRO-ED, 1993.

2) Leopold NA, Kagel MA: Prepharyngeal dysphagia in Parkinson's disease. Dysphagia, 1996, 11: 14-22. [Medline] [CrossRef]

3) Freed ML, Freed L, Chatburn RL, et al.: Electrical stimulation for swallowing disorders caused by stroke. Respir Care, 2001, 46: 466-474. [Medline]

4) Bastian RW: Videoendoscopic evaluation of patients with dysphagia: an adjunct to the modified barium swallow. Otolaryngol Head Neck Surg, 1991, 104: 339-350. [Medline]

5) Daniels SK: Optimal patterns of care for dysphagic stroke patients. Semin Speech Lang, 2000, 21: 323-331. [Medline] [CrossRef]

6) Kim NH, Kwon YS, Lee KH: A study on the nutritional status, symptoms, and information needs in stroke patients with dysphagia. Kor Soc Biolog Nurs Sci, 2011, 13: 72-80

7) Woo JH, Jung MW, Kim KY: The relevant factors and effect of swallowing function on oropharyngeal stimulation program in stroke patients with swallowing disorder. J Kor Acad Occup Ther, 2009, 17: 1-12.

8) Jung MW, Kim TH, Jung HY: Neuromuscular electrical stimulation of the neck muscles for eysphagia. J Kor Acad Occup Ther, 2005, 13: 15-23.

9) Shin SJ, Park JM, Lee DW: The effects of electrical stimulation on the neck muscles of patients with a swallowing disorder caused by a stroke. J Kor Acad Occup Ther, 2009, 17: 17-28.

10) Park CL, O’Nell PA, Martin DF: A pilot exploratory study of oral electrical stimulation on swallowing function following stoke: an innovative technique. Dysphagia, 1997, 12: 161-166. [Medline] [CrossRef]

11) Park SH, Song KS, Seo JH: Characteristics of motor evoked potential recording from swallowing muscles obtained by transcranial magnetic stimulation in healthy subjects. J Kor Acad Rehab Med, 2009, 33: 21-27. 